\title{
Quadratic Algebra Approach to an Exactly Solvable Position-Dependent Mass Schrödinger Equation in Two Dimensions
}

\author{
Christiane QUESNE
}

Physique Nucléaire Théorique et Physique Mathématique, Université Libre de Bruxelles, Campus de la Plaine CP229, Boulevard du Triomphe, B-1050 Brussels, Belgium

E-mail: cquesne@ulb.ac.be

Received March 30, 2007, in final form May 08, 2007; Published online May 17, 2007

Original article is available at http://www.emis.de/journals/SIGMA/2007/067/

\begin{abstract}
An exactly solvable position-dependent mass Schrödinger equation in two dimensions, depicting a particle moving in a semi-infinite layer, is re-examined in the light of recent theories describing superintegrable two-dimensional systems with integrals of motion that are quadratic functions of the momenta. To get the energy spectrum a quadratic algebra approach is used together with a realization in terms of deformed parafermionic oscillator operators. In this process, the importance of supplementing algebraic considerations with a proper treatment of boundary conditions for selecting physical wavefunctions is stressed. Some new results for matrix elements are derived. This example emphasizes the interest of a quadratic algebra approach to position-dependent mass Schrödinger equations.
\end{abstract}

Key words: Schrödinger equation; position-dependent mass; quadratic algebra

2000 Mathematics Subject Classification: 81R12; 81R15

\section{Introduction}

Quantum mechanical systems with a position-dependent (effective) mass (PDM) have attracted a lot of attention and inspired intense research activities during recent years. They are indeed very useful in the study of many physical problems, such as electronic properties of semiconductors [1] and quantum dots [2], nuclei [3], quantum liquids [4], ${ }^{3}$ He clusters [5], metal clusters [6], etc.

Looking for exact solutions of the Schrödinger equation with a PDM has become an interesting research topic because such solutions may provide a conceptual understanding of some physical phenomena, as well as a testing ground for some approximation schemes (for a list of references see, e.g., [7]). For such a purpose, use has been made of methods known in the constant-mass case and extended to a PDM context, such as point canonical transformations [8, 9, 10], Lie algebraic methods [11, 12, 13, 14], as well as supersymmetric quantum mechanical (SUSYQM) and shape-invariance techniques $[15,16]$.

Although mostly one-dimensional equations have been considered up to now, several works have recently paid attention to $d$-dimensional problems $[7,17,18,19,20,21,22]$. In [7] (henceforth referred to as I and whose equations will be quoted by their number preceded by I), we have analyzed $d$-dimensional PDM Schrödinger equations in the framework of first-order intertwining operators and shown that with a pair $\left(H, H_{1}\right)$ of intertwined Hamiltonians we can associate another pair $\left(R, R_{1}\right)$ of second-order partial differential operators related to the same intertwining operator and such that $H$ (resp. $H_{1}$ ) commutes with $R$ (resp. $R_{1}$ ). In the context of SUSYQM based on an sl(1/1) superalgebra, $R$ and $R_{1}$ can be interpreted as SUSY partners, while $H$ and $H_{1}$ are related to the Casimir operator of a larger gl(1/1) superalgebra. 
In the same work, we have also applied our general theory to an explicit example, depicting a particle moving in a two-dimensional semi-infinite layer. This model may be of interest in the study of quantum wires with an abrupt termination in an environment that can be modelled by a dependence of the carrier effective mass on the position. It illustrates the influence of a uniformity breaking in a quantum channel on the production of bound states, as it was previously observed in the case of a quantum dot or a bend [23, 24].

From a theoretical viewpoint, our model has proved interesting too because it is solvable in two different ways: by separation of variables in the corresponding Schrödinger equation or employing SUSYQM and shape-invariance techniques. The former method relies upon the existence of an integral of motion $L$, while, as above-mentioned, the latter is based on the use of $R$. In other words, the three second-order partial differential operators $H, L$ and $R$ form a set of algebraically independent integrals of motion, which means that the system is superintegrable.

Let us recall that in classical mechanics [25], an integrable system on a $d$-dimensional manifold is a system which has $d$ functionally independent (globally defined) integrals of motion in involution (including the Hamiltonian). Any system with more that $d$ functionally independent integrals of motion is called superintegrable. It is maximally superintegrable if it admits the maximum number $2 d-1$ of integrals of motion. The latter form a complete set so that any other integral of motion can be expressed in terms of them. In particular, the Poisson bracket of any two basic integrals, being again a constant of motion, can be written as a (in general) nonlinear function of them. Such results can be extended to quantum mechanics [26], so that for quantum counterparts of maximally superintegrable systems we get (in general) nonlinear associative algebras of algebraically independent observables, all of them commuting with $H$.

The simplest case corresponds to the class of two-dimensional superintegrable systems with integrals of motion that are linear and quadratic functions of the momenta. The study and classification of such systems, dating back to the 19th century and revived in the 1960ties [27, 28, 29], have recently been the subject of intense research activities and substantial progress has been made in this area (see [30, 31, 32, 33, 34, 35, 36, 37, 38, 39, 40, 41, 42, 43, 44, 45, 46, 47, 48] and references quoted therein). In particular, it has been shown that their integrals of motion generate a quadratic Poisson algebra (in the classical case) or a quadratic associative algebra (in the quantum one) with a Casimir of sixth degree in the momenta and the general form of these algebras has been uncovered [36, 45, 46, 47, 48]. Algebras of this kind have many similarities to the quadratic Racah algebra QR(3) (a special case of the quadratic Askey-Wilson algebra QAW(3)) [31,32]. They actually coincide with QR(3) whenever one of their parameters vanishes. The eigenvalues and eigenfunctions of the superintegrable system Hamiltonian can be found from the finite-dimensional irreducible representations of these algebras. The latter can be determined by a ladder-operator method [31, 32, 33, 34] or through a realization [35, 36] in terms of (generalized) deformed parafermionic operators [49], which are a finite-dimensional version of deformed oscillator operators [50].

Since our two-dimensional PDM model belongs to this class of superintegrable systems, it is interesting to analyze it in the light of such topical and innovative theories. This is one of the purposes of the present paper, which will therefore provide us with a third method for solving the PDM Schrödinger equation. In such a process, we will insist on the necessity of supplementing algebraic calculations with a proper treatment of the wavefunction boundary conditions imposed by the physics of the problem - a point that is not always highlighted enough.

Another purpose of this work is to stress the interest of a quadratic algebra approach to PDM Schrödinger equations. If the presence of such an algebra was already noted before in a one-dimensional example [51], this is indeed - as far as the author knows - the first case where an algebra of this kind is used as a tool for solving a physical problem in a PDM context.

This paper is organized as follows. In Section 2, the two-dimensional PDM model of I is briefly reviewed and some important comments on its mathematical structure are made in 
conjunction with the physics of the problem. In Section 3, a quadratic algebra associated with such a model is then introduced and its classical limit is obtained. The finite-dimensional irreducible representations of the algebra are determined in Section 4. Finally, Section 5 contains the conclusion.

\section{Exactly solvable and superintegrable PDM model in a two-dimensional semi-infinite layer}

In I we considered a particle moving in a two-dimensional semi-infinite layer of width $\pi / q$, parallel to the $x$-axis and with impenetrable barriers at the boundaries. The variables $x, y$ vary in the domain

$$
D: \quad 0<x<\infty, \quad-\frac{\pi}{2 q}<y<\frac{\pi}{2 q},
$$

and the wavefunctions have to satisfy the conditions

$$
\psi(0, y)=0, \quad \psi\left(x, \pm \frac{\pi}{2 q}\right)=0 .
$$

The mass of the particle is $m(x)=m_{0} M(x)$, where the dimensionless function $M(x)$ is given by

$$
M(x)=\operatorname{sech}^{2} q x .
$$

In units wherein $\hbar=2 m_{0}=1$, the Hamiltonian of the model can be written as

$$
H^{(k)}=-\partial_{x} \frac{1}{M(x)} \partial_{x}-\partial_{y} \frac{1}{M(x)} \partial_{y}+V_{\mathrm{eff}}^{(k)}(x),
$$

where we adopt the general form (I2.2) and

$$
V_{\mathrm{eff}}^{(k)}(x)=-q^{2} \cosh ^{2} q x+q^{2} k(k-1) \operatorname{csch}^{2} q x
$$

is an effective potential. This function includes some terms depending on the ambiguity parameters [52], which allow any ordering of the noncommutating momentum and PDM operators (see equation (I2.3)). In (2.4), the constant $k$ is assumed positive and we have set an irrelevant additive constant $v_{0}$ to zero.

As shown in I, both the operators

$$
L=-\partial_{y}^{2}
$$

and

$$
\begin{aligned}
R^{(k)}= & \eta^{(k) \dagger} \eta^{(k)} \\
= & -\cosh ^{2} q x \sin ^{2} q y \partial_{x}^{2}+2 \sinh q x \cosh q x \sin q y \cos q y \partial_{x y}^{2}-\sinh ^{2} q x \cos ^{2} q y \partial_{y}^{2} \\
& +q \sinh q x \cosh q x\left(1-4 \sin ^{2} q y\right) \partial_{x}+q\left(1+4 \sinh ^{2} q x\right) \sin q y \cos q y \partial_{y} \\
& +q^{2}\left(\sinh ^{2} q x-\sin ^{2} q y-3 \sinh ^{2} q x \sin ^{2} q y\right)-q^{2} k\left(1+\operatorname{csch}^{2} q x \sin ^{2} q y\right) \\
& +q^{2} k^{2} \operatorname{csch}^{2} q x \sin ^{2} q y,
\end{aligned}
$$

where

$$
\begin{aligned}
& \eta^{(k) \dagger}=-\cosh q x \sin q y \partial_{x}+\sinh q x \cos q y \partial_{y}-q \sinh q x \sin q y-q k \operatorname{csch} q x \sin q y, \\
& \eta^{(k)}=\cosh q x \sin q y \partial_{x}-\sinh q x \cos q y \partial_{y}+q \sinh q x \sin q y-q k \operatorname{csch} q x \sin q y,
\end{aligned}
$$

commute with $H^{(k)}$, although not with one another. Hence one may diagonalize either $H^{(k)}$ and $L$ or $H^{(k)}$ and $R^{(k)}$ simultaneously. This leads to two alternative bases for the Hamiltonian 
eigenfunctions, corresponding to the eigenvalues

$$
E_{N}^{(k)}=q^{2}(N+2)(N+2 k+1), \quad N=0,1,2, \ldots,
$$

with degeneracies

$$
\operatorname{deg}(N)=\left[\frac{N}{2}\right]+1
$$

where $[N / 2]$ stands for the integer part of $N / 2$.

The first basis is obtained by separating the variables $x, y$ in the PDM Schrödinger equation and its members, associated with the eigenvalues $(l+1)^{2} q^{2}$ of $L$, read

$$
\psi_{n, l}^{(k)}(x, y)=\phi_{n, l}^{(k)}(x) \chi_{l}(y), \quad n, l=0,1,2, \ldots,
$$

with $N=2 n+l$,

$$
\begin{aligned}
& \phi_{n, l}^{(k)}(x)=\mathcal{N}_{n, l}^{(k)}(\tanh q x)^{k}(\operatorname{sech} q x)^{l+2} P_{n}^{\left(k-\frac{1}{2}, l+1\right)}\left(1-2 \tanh ^{2} q x\right), \\
& \chi_{l}(y)= \begin{cases}\sqrt{\frac{2 q}{\pi}} \cos [(l+1) q y] & \text { for } l=0,2,4, \ldots, \\
\sqrt{\frac{2 q}{\pi}} \sin [(l+1) q y] & \text { for } l=1,3,5, \ldots,\end{cases}
\end{aligned}
$$

and $\mathcal{N}_{n, l}^{(k)}$ a normalization constant given in equation (I3.18).

The second basis, resulting from the intertwining relation

$$
\eta^{(k)} H^{(k)}=H_{1}^{(k)} \eta^{(k)}, \quad H_{1}^{(k)}=H^{(k+1)}+2 q^{2} k,
$$

and its Hermitian conjugate, can be built by successive applications of operators of type $\eta^{(k) \dagger}$,

$$
\Psi_{N, N_{0}}^{(k)}(x, y)=\overline{\mathcal{N}}_{N, N_{0}}^{(k)} \eta^{(k) \dagger} \eta^{(k+1) \dagger} \cdots \eta^{(k+\nu-1) \dagger} \Psi_{N_{0}, N_{0}}^{(k+\nu)}(x, y),
$$

on functions $\Psi_{N_{0}, N_{0}}^{(k+\nu)}(x, y)$, annihilated by $\eta^{(k+\nu)}$ and given in Eqs. (I3.28), (I3.32) and (I3.34). In (2.9), $N_{0}$ runs over $0,2,4, \ldots, N$ or $N-1$, according to whether $N$ is even or odd, while $\nu$, defined by $\nu=N-N_{0}$, determines the $R^{(k)}$ eigenvalue

$$
r_{\nu}^{(k)}=q^{2} \nu(\nu+2 k), \quad \nu=0,1,2, \ldots
$$

Although an explicit expression of the normalization coefficient $\overline{\mathcal{N}}_{N, N_{0}}^{(k)}$ is easily obtained (see equation (I3.41)), this is not the case for $\Psi_{N, N_{0}}^{(k)}(x, y)$ (except for some low values of $N$ and $N_{0}$ ), nor for the expansion of $\Psi_{N, N_{0}}^{(k)}(x, y)$ into the first basis eigenfunctions $\psi_{n, l}^{(k)}(x, y)$, which is given by rather awkward formulas (see equations (I3.46), (I3.51), (I3.55) and (I3.56)).

Before proceeding to a quadratic algebra approach to the problem in Section 3, it is worth making a few valuable observations, which were not included in I.

Mathematically speaking, the separable Schrödinger equation of our model admits four linearly independent solutions obtained by combining the two independent solutions of the secondorder differential equation in $x$ with those of the second-order differential equation in $y$. Among those four functions, only the combination $\psi_{n, l}^{(k)}(x, y)$, considered in $(2.7)$, satisfies all the boundary conditions and is normalizable on $D$. It is indeed clear that the alternative solution to the differential equation in $x$ is not normalizable, while that to the differential equation in $y$,

$$
\bar{\chi}_{l}(y) \propto \begin{cases}\sin [(l+1) q y] & \text { for } l=0,2,4, \ldots, \\ \cos [(l+1) q y] & \text { for } l=-1,1,3,5, \ldots\end{cases}
$$


violates the second condition in equation (2.1). Hence the three remaining combinations provide unphysical functions.

Some mathematical considerations might also lead to another choice than $L$ and $R^{(k)}$ for the basic integrals of motion complementing $H^{(k)}$. First of all, instead of $L$, one might select the operator $p_{y}=-\mathrm{i} \partial_{y}$, which obviously satisfies the condition $\left[H^{(k)}, p_{y}\right]=0$. This would result in a linear and a quadratic (in the momenta) integrals of motion, generating a much simpler quadratic algebra than that to be considered in Section 3. It should be realized, however, that the eigenfunctions $e^{\mathrm{i} m y}(m \in \mathbb{Z})$ of $p_{y}$, being linear combinations of the physical and unphysical functions (2.8) and (2.11), are useless from a physical viewpoint. We are therefore forced to consider the second-order operator $L$ instead of $p_{y}$.

Furthermore, it is straightforward to see that another pair of first-order differential operators

$$
\begin{aligned}
& \bar{\eta}^{(k) \dagger}=-\cosh q x \cos q y \partial_{x}-\sinh q x \sin q y \partial_{y}-q \sinh q x \cos q y-q k \operatorname{csch} q x \cos q y \\
& \bar{\eta}^{(k)}=\cosh q x \cos q y \partial_{x}+\sinh q x \sin q y \partial_{y}+q \sinh q x \cos q y-q k \operatorname{csch} q x \cos q y
\end{aligned}
$$

intertwines with $H^{(k)}$ and $H_{1}^{(k)}$, i.e., satisfies the relation

$$
\bar{\eta}^{(k)} H^{(k)}=H_{1}^{(k)} \bar{\eta}^{(k)}, \quad H_{1}^{(k)}=H^{(k+1)}+2 q^{2} k,
$$

and its Hermitian conjugate. Such operators correspond to the choice $a=c=g=0, b=d=1$ in equation (I2.29).

As a consequence of (2.14), the operator

$$
\begin{aligned}
\bar{R}^{(k)}= & \bar{\eta}^{(k) \dagger} \bar{\eta}^{(k)} \\
= & -\cosh ^{2} q x \cos ^{2} q y \partial_{x}^{2}-2 \sinh q x \cosh q x \sin q y \cos q y \partial_{x y}^{2}-\sinh ^{2} q x \sin ^{2} q y \partial_{y}^{2} \\
& +q \sinh q x \cosh q x\left(1-4 \cos ^{2} q y\right) \partial_{x}-q\left(1+4 \sinh ^{2} q x\right) \sin q y \cos q y \partial_{y} \\
& +q^{2}\left(\sinh ^{2} q x-\cos ^{2} q y-3 \sinh ^{2} q x \cos ^{2} q y\right)-q^{2} k\left(1+\operatorname{csch}^{2} q x \cos ^{2} q y\right) \\
& +q^{2} k^{2} \operatorname{csch}^{2} q x \cos ^{2} q y,
\end{aligned}
$$

commutes with $H^{(k)}$ and is therefore another integral of motion. It can of course be expressed in terms of $H^{(k)}, L$ and $R^{(k)}$, as it can be checked that

$$
H^{(k)}=L+R^{(k)}+\bar{R}^{(k)}+2 q^{2} k .
$$

However, we have now at our disposal three (dependent) integrals of motion $L, R^{(k)}$ and $\bar{R}^{(k)}$ in addition to $H^{(k)}$, so that we may ask the following question: what is the best choice for the basic integrals of motion from a physical viewpoint?

This problem is easily settled by noting that the zero modes of $\bar{\eta}^{(k)}$,

$$
\bar{\omega}_{s}^{(k)}(x, y)=(\tanh q x)^{k}(\operatorname{sech} q x)^{s+1}(\sin q y)^{s},
$$

violate the second condition in equation (2.1) for any real value of $s$ and therefore lead to unphysical functions. This contrasts with what happens for the zero modes $\omega_{s}^{(k)}(x, y)$ of $\eta^{(k)}$, given in (I3.28), which are physical functions for $s>0$ and can therefore be used to build the functions $\Psi_{N, N_{0}}^{(k)}(x, y)$ considered in (2.9), as it was shown in (I3.32). We conclude that the physics of the model imposes the choice of $L$ and $R^{(k)}$ as basic integrals of motion. 


\section{Quadratic associative algebra and its classical limit}

It has been shown $[36,47]$ that for any two-dimensional quantum superintegrable system with integrals of motion $A, B$, which are second-order differential operators, one can construct a quadratic associative algebra generated by $A, B$, and their commutator $C$. This operator is not independent of $A, B$, but since it is a third-order differential operator, it cannot be written as a polynomial function of them. The general form of the quadratic algebra commutation relations is

$$
\begin{aligned}
& {[A, B]=C,} \\
& {[A, C]=\alpha A^{2}+\gamma\{A, B\}+\delta A+\epsilon B+\zeta,} \\
& {[B, C]=a A^{2}-\gamma B^{2}-\alpha\{A, B\}+d A-\delta B+z .}
\end{aligned}
$$

Here $\{A, B\} \equiv A B+B A$,

$$
\begin{aligned}
& \delta=\delta(H)=\delta_{0}+\delta_{1} H, \quad \epsilon=\epsilon(H)=\epsilon_{0}+\epsilon_{1} H, \quad \zeta=\zeta(H)=\zeta_{0}+\zeta_{1} H+\zeta_{2} H^{2}, \\
& d=d(H)=d_{0}+d_{1} H, \quad z=z(H)=z_{0}+z_{1} H+z_{2} H^{2},
\end{aligned}
$$

and $\alpha, \gamma, a, \delta_{i}, \epsilon_{i}, \zeta_{i}, d_{i}, z_{i}$ are some constants. Note that it is the Jacobi identity $[A,[B, C]]=$ $[B,[A, C]]$ that imposes some relations between coefficients in (3.2) and (3.3).

Such a quadratic algebra closes at level 6 [47] or, in other words, it has a Casimir operator which is a sixth-order differential operator [36],

$$
\begin{aligned}
K= & C^{2}+\frac{2}{3} a A^{3}-\frac{1}{3} \alpha\{A, A, B\}-\frac{1}{3} \gamma\{A, B, B\}+\left(\frac{2}{3} \alpha^{2}+d+\frac{2}{3} a \gamma\right) A^{2} \\
& +\left(\frac{1}{3} \alpha \gamma-\delta\right)\{A, B\}+\left(\frac{2}{3} \gamma^{2}-\epsilon\right) B^{2}+\left(\frac{2}{3} \alpha \delta+\frac{1}{3} a \epsilon+\frac{1}{3} d \gamma+2 z\right) A \\
& +\left(-\frac{1}{3} \alpha \epsilon+\frac{2}{3} \gamma \delta-2 \zeta\right) B+\frac{1}{3} \gamma z-\frac{1}{3} \alpha \zeta \\
= & k_{0}+k_{1} H+k_{2} H^{2}+k_{3} H^{3},
\end{aligned}
$$

where $k_{i}$ are some constants and $\{A, B, C\} \equiv A B C+A C B+B A C+B C A+C A B+C B A$.

For our two-dimensional PDM model, described by the Hamiltonian defined in equations (2.2)-(2.4), we shall take

$$
A=R, \quad B=L,
$$

where, for simplicity's sake, we dropped the superscript $(k)$ because no confusion can arise outside the SUSYQM context.

To determine their commutation relations, it is worth noting first that their building blocks, the first-order differential operators $\partial_{y}, \eta^{\dagger}$ and $\eta$, generate another quadratic algebra together with the other set of intertwining operators $\bar{\eta}^{\dagger}, \bar{\eta}$, given in (2.12) and (2.13). Their commutation relations are indeed easily obtained as

$$
\begin{aligned}
& {\left[\partial_{y}, \eta\right]=q \bar{\eta}, \quad\left[\partial_{y}, \bar{\eta}\right]=-q \eta, \quad[\eta, \bar{\eta}]=q \partial_{y},} \\
& {\left[\eta, \eta^{\dagger}\right]=2 q^{2} k\left(1+\xi^{2}\right), \quad\left[\bar{\eta}, \bar{\eta}^{\dagger}\right]=2 q^{2} k\left(1+\bar{\xi}^{2}\right), \quad\left[\eta, \bar{\eta}^{\dagger}\right]=-q \partial_{y}+2 q^{2} k \xi \bar{\xi},}
\end{aligned}
$$

and their Hermitian conjugates. In (3.7), we have defined

$$
\xi=-(2 q k)^{-1}\left(\eta+\eta^{\dagger}\right)=\operatorname{csch} q x \sin q y, \quad \bar{\xi}=-(2 q k)^{-1}\left(\bar{\eta}+\bar{\eta}^{\dagger}\right)=\operatorname{csch} q x \cos q y .
$$

Interestingly, $\partial_{y}, \eta$ and $\bar{\eta}$ (as well as $\partial_{y}, \eta^{\dagger}$ and $\bar{\eta}^{\dagger}$ ) close an sl(2) subalgebra.

From these results, it is now straightforward to show that the operator $C$ in (3.1) is given by

$$
C=q\left\{\partial_{y}, \eta^{\dagger} \bar{\eta}+\bar{\eta}^{\dagger} \eta\right\}
$$


and that the coefficients in (3.2) and (3.3) are

$$
\begin{aligned}
& \alpha=\gamma=8 q^{2}, \quad \delta=8 q^{2}\left[q^{2}(2 k-1)-H\right], \quad \epsilon=16 q^{4}(k-1)(k+1), \\
& \zeta=8 q^{4}(k-1)\left(2 q^{2} k-H\right), \quad a=0, \quad d=16 q^{4}, \quad z=8 q^{4}\left(2 q^{2} k-H\right) .
\end{aligned}
$$

On inserting the latter in (3.4), we obtain for the value of the Casimir operator

$$
K=-4 q^{4}\left[2 q^{2}(7 k-6)-3 H\right]\left(2 q^{2} k-H\right) .
$$

It is worth noting that since $a=0$ in (3.3), we actually have here an example of quadratic Racah algebra $\mathrm{QR}(3)$ [31].

Before proceeding to a study of its finite-dimensional irreducible representations in Section 4, it is interesting to consider its classical limit. For such a purpose, since we have adopted units wherein $\hbar=2 m_{0}=1$, we have first to make a change of variables and of parameters restoring a dependence on $\hbar$ (but keeping $2 m_{0}=1$ for simplicity's sake) before letting $\hbar$ go to zero.

An appropriate transformation is

$$
X=\hbar x, \quad Y=\hbar y, \quad P_{X}=-\mathrm{i} \hbar \partial_{X}, \quad P_{Y}=-\mathrm{i} \hbar \partial_{Y}, \quad Q=\frac{q}{\hbar}, \quad K=\hbar k .
$$

On performing it on the Hamiltonian given in equations (2.2)-(2.4), we obtain

$$
H=-\hbar^{2}\left(\partial_{X} \cosh ^{2} Q X \partial_{X}+\partial_{Y} \cosh ^{2} Q X \partial_{Y}\right)-\hbar^{2} Q^{2} \cosh ^{2} Q X+Q^{2} K(K-\hbar) \operatorname{csch}^{2} Q X,
$$

yielding the classical Hamiltonian

$$
H_{\mathrm{c}}=\lim _{\hbar \rightarrow 0} H=\cosh ^{2} Q X\left(P_{X}^{2}+P_{Y}^{2}\right)+Q^{2} K^{2} \operatorname{csch}^{2} Q X
$$

A similar procedure applied to the intertwining operators leads to

$$
\begin{aligned}
& \eta_{\mathrm{c}}=\lim _{\hbar \rightarrow 0} \eta=\mathrm{i} \cosh Q X \sin Q Y P_{X}-\mathrm{i} \sinh Q X \cos Q Y P_{Y}-Q K \operatorname{csch} Q X \sin Q Y, \\
& \bar{\eta}_{\mathrm{c}}=\lim _{\hbar \rightarrow 0} \bar{\eta}=\mathrm{i} \cosh Q X \cos Q Y P_{X}+\mathrm{i} \sinh Q X \sin Q Y P_{Y}-Q K \operatorname{csch} Q X \cos Q Y,
\end{aligned}
$$

together with $\eta_{\mathrm{c}}^{*}=\lim _{\hbar \rightarrow 0} \eta^{\dagger}$ and $\bar{\eta}_{\mathrm{c}}^{*}=\lim _{\hbar \rightarrow 0} \bar{\eta}^{\dagger}$, while the operators quadratic in the momenta give rise to the functions

$$
\begin{aligned}
L_{\mathrm{c}}=\lim _{\hbar \rightarrow 0} L= & P_{Y}^{2}, \\
R_{\mathrm{c}}=\lim _{\hbar \rightarrow 0} R= & \cosh ^{2} Q X \sin ^{2} Q Y P_{X}^{2}-2 \sinh Q X \cosh Q X \sin Q Y \cos Q Y P_{X} P_{Y} \\
& +\sinh ^{2} Q X \cos ^{2} Q Y P_{Y}^{2}+Q^{2} K^{2} \operatorname{csch}^{2} Q X \sin ^{2} Q Y, \\
\bar{R}_{\mathrm{c}}=\lim _{\hbar \rightarrow 0} \bar{R}= & \cosh ^{2} Q X \cos ^{2} Q Y P_{X}^{2}+2 \sinh Q X \cosh Q X \sin Q Y \cos Q Y P_{X} P_{Y} \\
& +\sinh ^{2} Q X \sin ^{2} Q Y P_{Y}^{2}+Q^{2} K^{2} \operatorname{csch}^{2} Q X \cos ^{2} Q Y,
\end{aligned}
$$

satisfying the relation

$$
H_{\mathrm{c}}=L_{\mathrm{c}}+R_{\mathrm{c}}+\bar{R}_{\mathrm{c}}
$$

The quadratic associative algebra (3.1)-(3.4) is now changed into a quadratic Poisson algebra, whose defining relations can be determined either by taking the $\operatorname{limit} \lim _{\hbar \rightarrow 0}(\mathrm{i} \hbar)^{-1}\left[O, O^{\prime}\right]=$ $\left\{O_{\mathrm{c}}, O_{\mathrm{c}}^{\prime}\right\}_{\mathrm{P}}$ or by direct calculation of the Poisson brackets $\left\{O_{\mathrm{c}}, O_{\mathrm{c}}^{\prime}\right\}_{\mathrm{P}}$ :

$$
\left\{A_{\mathrm{c}}, B_{\mathrm{c}}\right\}_{\mathrm{P}}=C_{\mathrm{c}},
$$




$$
\begin{aligned}
& \left\{A_{\mathrm{c}}, C_{\mathrm{c}}\right\}_{\mathrm{P}}=\alpha_{\mathrm{c}} A_{\mathrm{c}}^{2}+2 \gamma_{\mathrm{c}} A_{\mathrm{c}} B_{\mathrm{c}}+\delta_{\mathrm{c}} A_{\mathrm{c}}+\epsilon_{\mathrm{c}} B_{\mathrm{c}}+\zeta_{\mathrm{c}}, \\
& \left\{B_{\mathrm{c}}, C_{\mathrm{c}}\right\}_{\mathrm{P}}=a_{\mathrm{c}} A_{\mathrm{c}}^{2}-\gamma_{\mathrm{c}} B_{\mathrm{c}}^{2}-2 \alpha_{\mathrm{c}} A_{\mathrm{c}} B_{\mathrm{c}}+d_{\mathrm{c}} A_{\mathrm{c}}-\delta_{\mathrm{c}} B_{\mathrm{c}}+z_{\mathrm{c}} .
\end{aligned}
$$

Here

$$
C_{\mathrm{c}}=\lim _{\hbar \rightarrow 0} \frac{C}{\mathrm{i} \hbar}=2 Q P_{Y}\left(\eta_{\mathrm{c}}^{*} \bar{\eta}_{\mathrm{c}}+\bar{\eta}_{\mathrm{c}}^{*} \eta_{\mathrm{c}}\right)
$$

and

$$
\alpha_{\mathrm{c}}=\gamma_{\mathrm{c}}=-8 Q^{2}, \quad \delta_{\mathrm{c}}=8 Q^{2} H_{\mathrm{c}}, \quad \epsilon_{\mathrm{c}}=-16 Q^{4} K^{2}, \quad \zeta_{\mathrm{c}}=a_{\mathrm{c}}=d_{\mathrm{c}}=z_{\mathrm{c}}=0 .
$$

Such a Poisson algebra has a vanishing Casimir:

$$
K_{\mathrm{c}}=\lim _{\hbar \rightarrow 0} K=0 .
$$

\section{Finite-dimensional irreducible representations of the quadratic associative algebra}

The quadratic algebra (3.1)-(3.4) can be realized in terms of (generalized) deformed oscillator operators $\mathcal{N}, b^{\dagger}, b$, satisfying the relations [50]

$$
\left[\mathcal{N}, b^{\dagger}\right]=b^{\dagger}, \quad[\mathcal{N}, b]=-b, \quad b^{\dagger} b=\Phi(\mathcal{N}), \quad b b^{\dagger}=\Phi(\mathcal{N}+1),
$$

where the structure function $\Phi(x)$ is a 'well-behaved' real function such that

$$
\Phi(0)=0, \quad \Phi(x)>0 \quad \text { for } \quad x>0 .
$$

This deformed oscillator algebra has a Fock-type representation, whose basis states $|m\rangle, m=0$, $1,2, \ldots,{ }^{1}$ fulfil the relations

$$
\begin{aligned}
& \mathcal{N}|m\rangle=m|m\rangle, \\
& b^{\dagger}|m\rangle=\sqrt{\Phi(m+1)}|m+1\rangle, \quad m=0,1,2, \ldots, \\
& b|0\rangle=0, \\
& b|m\rangle=\sqrt{\Phi(m)}|m-1\rangle, \quad m=1,2, \ldots
\end{aligned}
$$

We shall be more specifically interested here in a subclass of deformed oscillator operators, which have a $(p+1)$-dimensional Fock space, spanned by $|p, m\rangle \equiv|m\rangle, m=0,1, \ldots, p$, due to the following property

$$
\Phi(p+1)=0
$$

of the structure function, implying that

$$
\left(b^{\dagger}\right)^{p+1}=b^{p+1}=0 .
$$

These are so-called (generalized) deformed parafermionic oscillator operators of order $p$ [49]. The general form of their structure function is given by

$$
\Phi(x)=x(p+1-x)\left(a_{0}+a_{1} x+a_{2} x^{2}+\cdots+a_{p-1} x^{p-1}\right),
$$

\footnotetext{
${ }^{1}$ We adopt here the unusual notation $|m\rangle$ in order to avoid confusion between the number of deformed bosons and the quantum number $n$ introduced in (2.7).
} 
where $a_{0}, a_{1}, \ldots, a_{p-1}$ may be any real constants such that the second condition in (4.1) is satisfied for $x=1,2, \ldots, p$.

A realization of the quadratic algebra (3.1)-(3.4) in terms of deformed oscillator operators $\mathcal{N}, b^{\dagger}, b$ reads $[36]$

$$
\begin{aligned}
& A=A(\mathcal{N}), \\
& B=\sigma(\mathcal{N})+b^{\dagger} \rho(\mathcal{N})+\rho(\mathcal{N}) b,
\end{aligned}
$$

where $A(\mathcal{N}), \sigma(\mathcal{N})$ and $\rho(\mathcal{N})$ are some functions of $\mathcal{N}$, which, in the $\gamma \neq 0$ case, are given by

$$
\begin{aligned}
& A(\mathcal{N})=\frac{\gamma}{2}\left[(\mathcal{N}+u)^{2}-\frac{1}{4}-\frac{\epsilon}{\gamma^{2}}\right] \\
& \sigma(\mathcal{N})=-\frac{\alpha}{4}\left[(\mathcal{N}+u)^{2}-\frac{1}{4}\right]+\frac{\alpha \epsilon-\gamma \delta}{2 \gamma^{2}}-\frac{\alpha \epsilon^{2}-2 \gamma \delta \epsilon+4 \gamma^{2} \zeta}{4 \gamma^{4}} \frac{1}{(\mathcal{N}+u)^{2}-\frac{1}{4}}, \\
& \rho^{2}(\mathcal{N})=\frac{1}{3 \cdot 2^{12} \gamma^{8}(\mathcal{N}+u)(\mathcal{N}+u+1)[2(\mathcal{N}+u)+1]^{2}}
\end{aligned}
$$

with the structure function

$$
\begin{aligned}
\Phi(x)= & -3072 \gamma^{6} K[2(\mathcal{N}+u)-1]^{2} \\
& -48 \gamma^{6}\left(\alpha^{2} \epsilon-\alpha \gamma \delta+a \gamma \epsilon-d \gamma^{2}\right)[2(\mathcal{N}+u)-3][2(\mathcal{N}+u)-1]^{4}[2(\mathcal{N}+u)+1] \\
& +\gamma^{8}\left(3 \alpha^{2}+4 a \gamma\right)[2(\mathcal{N}+u)-3]^{2}[2(\mathcal{N}+u)-1]^{4}[2(\mathcal{N}+u)+1]^{2} \\
& +768\left(\alpha \epsilon^{2}-2 \gamma \delta \epsilon+4 \gamma^{2} \zeta\right)^{2} \\
& +32 \gamma^{4}\left(3 \alpha^{2} \epsilon^{2}-6 \alpha \gamma \delta \epsilon+2 a \gamma \epsilon^{2}+2 \gamma^{2} \delta^{2}-4 d \gamma^{2} \epsilon+8 \gamma^{3} z+4 \alpha \gamma^{2} \zeta\right) \\
& \times[2(\mathcal{N}+u)-1]^{2}\left[12(\mathcal{N}+u)^{2}-12(\mathcal{N}+u)-1\right] \\
& -256 \gamma^{2}\left(3 \alpha^{2} \epsilon^{3}-9 \alpha \gamma \delta \epsilon^{2}+a \gamma \epsilon^{3}+6 \gamma^{2} \delta^{2} \epsilon-3 d \gamma^{2} \epsilon^{2}+2 \gamma^{4} \delta^{2}+2 d \gamma^{4} \epsilon+12 \gamma^{3} \epsilon z\right. \\
& \left.-4 \gamma^{5} z+12 \alpha \gamma^{2} \epsilon \zeta-12 \gamma^{3} \delta \zeta+4 \alpha \gamma^{4} \zeta\right)[2(\mathcal{N}+u)-1]^{2} .
\end{aligned}
$$

These functions depend upon two (so far undetermined) constants, $u$ and the eigenvalue of the Casimir operator $K$ (which we denote by the same symbol).

Such a realization is convenient to determine the representations of the quadratic algebra in a basis wherein the generator $A$ is diagonal together with $K$ (or, equivalently, $H$ ) because the former is already diagonal with eigenvalues $A(m)$. The $(p+1)$-dimensional representations, associated with $(p+1)$-fold degenerate energy levels, correspond to the restriction to deformed parafermionic operators of order $p$ [36]. The first condition in (4.1) can then be used with equation (4.3) to compute $u$ and $K$ (or $E$ ) in terms of $p$ and of the Hamiltonian parameters. A choice is then made between the various solutions that emerge from the calculations by imposing the second restriction in $(4.1)$ for $x=1,2, \ldots, p$.

In the present case, for the set of parameters (3.8), the complicated structure function (4.9) drastically simplifies to yield the factorized expression

$$
\begin{aligned}
\Phi(x)= & 3 \cdot 2^{30} q^{20}(2 x+2 u+k-1)(2 x+2 u+k-2)(2 x+2 u-k)(2 x+2 u-k-1) \\
& \times\left(2 x+2 u-\frac{1}{2}+\Delta\right)\left(2 x+2 u-\frac{3}{2}+\Delta\right)\left(2 x+2 u-\frac{1}{2}-\Delta\right)\left(2 x+2 u-\frac{3}{2}-\Delta\right),
\end{aligned}
$$

where

$$
\Delta=\sqrt{\left(k-\frac{1}{2}\right)^{2}+\frac{E}{q^{2}}} .
$$


Furthermore, the eigenvalues of the operator $A$ become

$$
A(m)=q^{2}(2 m+2 u-k)(2 m+2 u+k) .
$$

Since $A=R$ is a positive-definite operator, only values of $u$ such that $A(m) \geq 0$ for $m=0$, $1, \ldots, p$ should be retained.

On taking this into account, the first condition in (4.1) can be satisfied by choosing either $u=k / 2$ or $u=(k+1) / 2$, yielding

$$
A(m)=4 q^{2} m(m+k)
$$

or

$$
A(m)=4 q^{2}\left(m+\frac{1}{2}\right)\left(m+k+\frac{1}{2}\right),
$$

respectively. For $u=k / 2$, equation (4.3), together with the second condition in (4.1), can be fulfilled in two different ways corresponding to $\Delta=2 p+k+1 \pm \frac{1}{2}$ or

$$
E=q^{2}\left(2 p+\frac{3}{2} \pm \frac{1}{2}\right)\left(2 p+2 k+\frac{1}{2} \pm \frac{1}{2}\right) .
$$

The resulting structure function reads

$$
\begin{aligned}
\Phi(x)= & 3 \cdot 2^{38} q^{20} x(p+1-x)\left(x-\frac{1}{2}\right)\left(p+1 \pm \frac{1}{2}-x\right)\left(x+k-\frac{1}{2}\right)(x+k-1) \\
& \times\left(x+p+k+\frac{1}{4} \pm \frac{1}{4}\right)\left(x+p+k-\frac{1}{4} \pm \frac{1}{4}\right) .
\end{aligned}
$$

Similarly, for $u=(k+1) / 2$, we obtain

$$
E=q^{2}\left(2 p+\frac{5}{2} \pm \frac{1}{2}\right)\left(2 p+2 k+\frac{3}{2} \pm \frac{1}{2}\right)
$$

and

$$
\begin{aligned}
\Phi(x)= & 3 \cdot 2^{38} q^{20} x(p+1-x)\left(x+\frac{1}{2}\right)\left(p+1 \pm \frac{1}{2}-x\right)(x+k)\left(x+k-\frac{1}{2}\right) \\
& \times\left(x+p+k+\frac{5}{4} \pm \frac{1}{4}\right)\left(x+p+k+\frac{3}{4} \pm \frac{1}{4}\right) .
\end{aligned}
$$

Our quadratic algebra approach has therefore provided us with a purely algebraic derivation of the eigenvalues of $H$ and $R$ in a basis wherein they are simultaneously diagonal. It now remains to see to which eigenvalues we can associate physical wavefunctions, i.e., normalizable functions satisfying equation (2.1). This will imply a correspondence between $|p, m\rangle$ and the functions $\Psi_{N, N-\nu}(x, y)$, defined in (2.9).

On comparing $A(m)$ to the known (physical) eigenvalues $r_{\nu}$ of $R$, given in (2.10), we note that the first choice (4.10) for $A(m)$ corresponds to even $\nu=2 m$ (hence to even $N$ ), while the second choice (4.11) is associated with odd $\nu=2 m+1$ (hence with odd $N$ ). Appropriate values of $p$ leading to the level degeneracies (2.6) are therefore $p=N / 2$ and $p=(N-1) / 2$, respectively. With this identification, both equations (4.12) and (4.14) yield the same result

$$
E=q^{2}\left(N+\frac{3}{2} \pm \frac{1}{2}\right)\left(N+2 k+\frac{1}{2} \pm \frac{1}{2}\right) .
$$

Comparison with (2.5) shows that only the upper sign choice in (4.16) leads to physical wavefunctions $\Psi_{N, N-\nu}(x, y)$.

Restricting ourselves to such a choice, we can now rewrite all the results obtained in this section in terms of $N$ and $\nu$ instead of $p$ and $m$. In particular, the two expressions (4.13) and (4.15) for the structure function can be recast in a single form $\Phi(m) \rightarrow \Phi_{\nu}$, where

$$
\begin{aligned}
\Phi_{\nu}= & 3 \cdot 2^{30} q^{20} \nu(\nu-1)(\nu+2 k-1)(\nu+2 k-2)(N+\nu+2 k)(N+\nu+2 k+1) \\
& \times(N-\nu+2)(N-\nu+3) .
\end{aligned}
$$


More importantly, our quadratic algebra analysis provides us with an entirely new result, namely the matrix elements of the integral of motion $L$ in the basis wherein $H$ and $R$ are simultaneously diagonal. On using indeed the correspondence $|p, m\rangle \rightarrow \Psi_{N, N-\nu}$, as well as the results in equations (4.2), (4.5), (4.7), (4.8) and (4.17), we obtain

$$
L \Psi_{N, N-\nu}=\sigma_{\nu} \Psi_{N, N-\nu}+\tau_{\nu} \Psi_{N, N-\nu+2}+\tau_{\nu+2} \Psi_{N, N-\nu-2}
$$

where we have reset $\sigma(m) \rightarrow \sigma_{\nu}, \rho(m) \rightarrow \rho_{\nu}$ and defined $\tau_{\nu}=s_{\nu} \rho_{\nu-2} \sqrt{\Phi_{\nu}}$. The explicit form of the coefficients on the right-hand side of (4.18) is given by

$$
\begin{aligned}
\sigma_{\nu}= & \frac{q^{2}}{2(\nu+k-1)(\nu+k+1)}\left\{-(\nu+k-1)^{2}(\nu+k+1)^{2}\right. \\
& +\left[N^{2}+(2 k+3) N+2 k^{2}+2 k+1\right](\nu+k-1)(\nu+k+1) \\
& -k(k-1)(N+k+1)(N+k+2)\}, \\
\tau_{\nu}^{2}= & \frac{q^{4}}{16(\nu+k-2)(\nu+k-1)^{2}(\nu+k)} \nu(\nu-1)(\nu+2 k-1)(\nu+2 k-2) \\
& \times(N-\nu+2)(N-\nu+3)(N+\nu+2 k)(N+\nu+2 k+1) .
\end{aligned}
$$

Note that $\tau_{\nu}$ is determined up to some phase factor $s_{\nu}$ depending on the convention adopted for the relative phases of $\Psi_{N, N-\nu}$ and $\Psi_{N, N-\nu+2}$.

For $N=4$, for instance, $\nu$ runs over 0,2 , 4, so that equations (4.18)-(4.20) become

$$
\begin{aligned}
L \Psi_{4,0}= & \frac{q^{2}}{k+3}\left[(13 k+21) \Psi_{4,0}+3 s_{4} \sqrt{\frac{2(k+1)(2 k+3)(2 k+9)}{k+2}} \Psi_{4,2}\right], \\
L \Psi_{4,2}= & q^{2}\left[\frac{3 s_{4}}{k+3} \sqrt{\frac{2(k+1)(2 k+3)(2 k+9)}{k+2}} \Psi_{4,0}+\frac{17 k^{2}+76 k+39}{(k+1)(k+3)} \Psi_{4,2}\right. \\
& \left.+\frac{s_{2}}{k+1} \sqrt{\frac{10(k+3)(2 k+1)(2 k+7)}{k+2}} \Psi_{4,4}\right] \\
L \Psi_{4,4}= & \frac{q^{2}}{k+1}\left[s_{2} \sqrt{\frac{10(k+3)(2 k+1)(2 k+7)}{k+2}} \Psi_{4,2}+5(k+3) \Psi_{4,4}\right] .
\end{aligned}
$$

As a check, these results can be compared with those derived from the action of $L$ on the expansions of $\Psi_{4,0}, \Psi_{4,2}$ and $\Psi_{4,4}$ in terms of the first basis eigenfunctions $\psi_{0,4}, \psi_{1,2}$ and $\psi_{2,0}$ (see, e.g., equations (I3.61) and (I3.49) for $\Psi_{4,0}$ and $\Psi_{4,4}$, respectively). This leads to the phase factors $s_{2}=s_{4}=-1$.

To conclude, it is worth mentioning that had we made the opposite choice in equation (3.5), i.e., $A=L$ and $B=R$, we would not have been able to use the deformed parafermionic realization (4.4), (4.5) to determine the energy spectrum. The counterpart of the parafermionic vacuum state would indeed have been a function annihilated by $L$ and therefore constructed from the unphysical function $\bar{\chi}_{-1}(y)$ of equation $(2.11)$.

\section{Conclusion}

In this paper, we have revisited the exactly solvable PDM model in a two-dimensional semiinfinite layer introduced in I. Here we have taken advantage of its superintegrability with two integrals of motion $L$ and $R$ that are quadratic in the momenta to propose a third method of solution in the line of some recent analyses of such problems. 
We have first determined the explicit form of the quadratic associative algebra generated by $L, R$ and their commutator. We have shown that it is a quadratic Racah algebra $\mathrm{QR}(3)$ and that its Casimir operator $K$ is a second-degree polynomial in $H$. We have also obtained the quadratic Poisson algebra arising in the classical limit.

We have then studied the finite-dimensional irreducible representations of our algebra in a basis wherein $K$ (or $H$ ) and $R$ are diagonal. For such a purpose, we have used a simple procedure, proposed in [36], consisting in mapping this basis onto deformed parafermionic oscillator states of order $p$. Among the results so obtained for the energy spectrum, we have selected those with which physical wavefunctions can be associated. This has illustrated once again the well-known fact that in quantum mechanics the physics is determined not only by algebraic properties of operators, but also by the boundary conditions imposed on wavefunctions. Our analysis has provided us with an interesting new result, not obtainable in general form in the SUSYQM approach of I, namely the matrix elements of $L$ in the basis wherein $H$ and $R$ are simultaneously diagonal.

As final points, it is worth observing that the approaches followed here are not the only ones available. First, one could have used a gauge transformation to relate equation (2.3) to a well-known superintegrable system in a Darboux space $([38,48]$ and references quoted therein). Second, the irreducible representations of $\mathrm{QR}(3)$ could have been constructed by the ladder-operator method employed in [31, 32, 33, 34]. This would have allowed us to express the transformation matrix elements between the bases $\psi_{n, l}^{(k)}$ and $\Psi_{N, N_{0}}^{(k)}\left(\right.$ denoted by $Z_{N_{0} ; n, l}^{(k)}$ in I) in terms of Racah-Wilson polynomials.

\section{References}

[1] Bastard G., Wave mechanics applied to semiconductor heterostructures, Editions de Physique, Les Ulis, 1988.

[2] Serra L., Lipparini E., Spin response of unpolarized quantum dots, Europhys. Lett. 40 (1997), 667-672.

[3] Ring P., Schuck P., The nuclear many body problem, Springer, New York, 1980.

[4] Arias de Saavedra F., Boronat J., Polls A., Fabrocini A., Effective mass of one ${ }^{4}$ He atom in liquid ${ }^{3}$ He, Phys. Rev. B 50 (1994), 4248-4251, cond-mat/9403075.

[5] Barranco M., Pi M., Gatica S.M., Hernández E.S., Navarro J., Structure and energetics of mixed ${ }^{4} \mathrm{He}-{ }^{3} \mathrm{He}$ drops, Phys. Rev. B 56 (1997), 8997-9003.

[6] Puente A., Serra Ll., Casas M., Dipole excitation of Na clusters with a non-local energy density functional, Z. Phys. D 31 (1994), 283-286.

[7] Quesne C., First-order intertwining operators and position-dependent mass Schrödinger equations in $d$ dimensions, Ann. Physics 321 (2006), 1221-1239, quant-ph/0508216.

[8] Bhattacharjie A., Sudarshan E.C.G., A class of solvable potentials, Nuovo Cimento 25 (1962), 864-879.

[9] Natanzon G.A., General properties of potentials for which the Schrödinger equation can be solved by means of hypergeometric functions, Theoret. and Math. Phys. 38 (1979), 146-153.

[10] Lévai G., A search for shape-invariant solvable potentials, J. Phys. A: Math. Gen. 22 (1989), 689-702.

[11] Alhassid Y., Gürsey F., Iachello F., Group theory approach to scattering. II. The Euclidean connection, Ann. Physics 167 (1986), 181-200.

[12] Wu J., Alhassid Y., The potential group approach and hypergeometric differential equations, J. Math. Phys. 31 (1990), 557-562.

[13] Englefield M.J., Quesne C., Dynamical potential algebras for Gendenshtein and Morse potentials, J. Phys. A: Math. Gen. 24 (1991), 3557-3574.

[14] Lévai G., Solvable potentials associated with su(1,1) algebras: a systematic study, J. Phys. A: Math. Gen. 27 (1994), 3809-3828.

[15] Cooper F., Khare A., Sukhatme U., Supersymmetry and quantum mechanics, Phys. Rep. 251 (1995), 267385, hep-th/9405029. 
[16] Bagchi B., Supersymmetry in quantum and classical mechanics, Chapman and Hall/CRC, Boca Raton, FL, 2000 .

[17] Chen G., Chen Z., Exact solutions of the position-dependent mass Schrödinger equation in $D$ dimensions, Phys. Lett. A 331 (2004), 312-315.

[18] Dong S.-H., Lozada-Cassou M., Exact solutions of the Schrödinger equation with the position-dependent mass for a hard-core potential, Phys. Lett. A 337 (2005), 313-320.

[19] Mustafa O., Mazharimousavi S.H., $d$-dimensional generalization of the point canonical transformation for a quantum particle with position-dependent mass, J. Phys. A: Math. Gen. 39 (2006), 10537-10547, math-ph/0602044.

[20] Mustafa O., Mazharimousavi S.H., Quantum particles trapped in a position-dependent mass barrier; a $d$ dimensional recipe, Phys. Lett. A 358 (2006), 259-261, quant-ph/0603134.

[21] Ju G.-X., Xiang Y., Ren Z.-Z., The localization of $s$-wave and quantum effective potential of a quasi-free particle with position-dependent mass, quant-ph/0601005.

[22] Gönül B., Koçak M., Explicit solutions for $N$-dimensional Schrödinger equations with position-dependent mass, J. Math. Phys. 47 (2006), 102101, 6 pages, quant-ph/0512035.

[23] Olendski O., Mikhailovska L., Bound-state evolution in curved waveguides and quantum wires, Phys. Rev. $B 66$ (2002), 035331, 8 pages.

[24] Gudmundsson V., Tang C.-S., Manolescu A., Bound state with negative binding energy induced by coherent transport in a two-dimensional quantum wire, Phys. Rev. B 72 (2005), 153306, 4 pages, cond-mat/0506009.

[25] Goldstein H., Classical mechanics, Addison-Wesley, Reading, MA, 1980.

[26] Dirac P.A.M., The principles of quantum mechanics, Oxford University Press, Oxford, 1981.

[27] Friš I., Mandrosov V., Smorodinsky Ya.A., Uhlir M., Winternitz P., On higher symmetries in quantum mechanics, Phys. Lett. 16 (1965), 354-356.

[28] Winternitz P., Smorodinsky Ya.A., Uhlir M., Friš I., Symmetry groups in classical and quantum mechanics, Sov. J. Nucl. Phys. 4 (1967), 444-450.

[29] Makharov A.A., Smorodinsky Ya.A., Valiev Kh., Winternitz P., A systematic search for nonrelativistic systems with dynamical symmetries. Part I: the integrals of motion, Nuovo Cimento A 52 (1967), 10611084.

[30] Hietarinta J., Direct methods for the search of the second invariant, Phys. Rep. 147 (1987), 87-154.

[31] Granovskii Ya.I., Lutzenko I.M., Zhedanov A.S., Mutual integrability, quadratic algebras, and dynamical symmetry, Ann. Physics 217 (1992), 1-20.

[32] Zhedanov A.S., "Hidden symmetry" of Askey-Wilson polynomials, Theoret. and Math. Phys. 89 (1991), $1146-1157$.

[33] Granovskii Ya.I., Zhedanov A.S., Lutsenko I.M., Quadratic algebras and dynamics in curved spaces. I. Oscillator, Theoret. and Math. Phys. 91 (1992), 474-480.

[34] Granovskii Ya.I., Zhedanov A.S., Lutsenko I.M., Quadratic algebras and dynamics in curved spaces. II. The Kepler problem, Theoret. and Math. Phys. 91 (1992), 604-612.

[35] Bonatsos D., Daskaloyannis C., Kokkotas K., Deformed oscillator algebras for two-dimensional quantum superintegrable systems, Phys. Rev. A 50 (1994), 3700-3709, hep-th/9309088.

[36] Daskaloyannis C., Quadratic Poisson algebras of two-dimensional classical superintegrable systems and quadratic associative algebras of quantum superintegrable systems, J. Math. Phys. 42 (2001), 1100-1119, math-ph/0003017.

[37] Daskaloyannis C., Ypsilantis K., Unified treatment and classification of superintegrable systems with integrals quadratic in momenta on a two-dimensional manifold, J. Math. Phys. 47 (2006), 042904, 38 pages, math-ph/0412055.

[38] Daskaloyannis C., Tanoudes Y., Classification of quantum superintegrable systems with quadratic integrals on two dimensional manifolds, math-ph/0607058.

[39] Létourneau P., Vinet L., Superintegrable systems: polynomial algebras and quasi-exactly solvable Hamiltonians, Ann. Physics 243 (1995), 144-168.

[40] Rañada M.F., Superintegrable $n=2$ systems, quadratic constants of motion, and potentials of Drach, J. Math. Phys. 38 (1997), 4165-4178. 
[41] Rañada M.F., Santander M., Superintegrable systems on the two-dimensional sphere $S^{2}$ and the hyperbolic plane $H^{2}$, J. Math. Phys. 40 (1999), 5026-5057.

[42] Tempesta P., Turbiner A.V., Winternitz P., Exact solvability of superintegrable systems, J. Math. Phys. 42 (2001), 4248-4257, hep-th/0011209.

[43] Kalnins E.G., Miller W.Jr., Pogosyan G.S., Superintegrability on the two-dimensional hyperboloid, J. Math. Phys. 38 (1997), 5416-5433.

[44] Kalnins E.G., Miller W.Jr., Hakobyan Y.M., Pogosyan G.S., Superintegrability on the two-dimensional hyperboloid. II, J. Math. Phys. 40 (1999), 2291-2306, quant-ph/9907037.

[45] Kalnins E.G., Kress J.M., Miller W.Jr., Second-order superintegrable systems in conformally flat spaces. I. Two-dimensional classical structure theory, J. Math. Phys. 46 (2005), 053509, 28 pages.

[46] Kalnins E.G., Kress J.M., Miller W.Jr., Second-order superintegrable systems in conformally flat spaces. II. The classical two-dimensional Stäckel transform, J. Math. Phys. 46 (2005), 053510, 15 pages.

[47] Kalnins E.G., Kress J.M., Miller W.Jr., Second-order superintegrable systems in conformally flat spaces. V. Two- and three-dimensional quantum systems, J. Math. Phys. 47 (2006), 093501, 25 pages.

[48] Kalnins E.G., Kress J.M., Miller W.Jr., Nondegenerate 2D complex Euclidean superintegrable systems and algebraic varieties, J. Phys. A: Math. Theor. 40 (2007), 3399-3411.

[49] Quesne C., Generalized deformed parafermions, nonlinear deformations of so(3) and exactly solvable potentials, Phys. Lett. A 193 (1994), 245-250.

[50] Daskaloyannis C., Generalized deformed oscillator and nonlinear algebras, J. Phys. A: Math. Gen. 24 (1991), L789-L794.

[51] Roy B., Roy P., Effective mass Schrödinger equation and nonlinear algebras, Phys. Lett. A 340 (2005), 70-73.

[52] von Roos O., Position-dependent effective masses in semiconductor theory, Phys. Rev. B 27 (1983), $7547-$ 7552 . 